Малиева Ф.Ф Об устойчивости разностной схемы с весами для уравнения теплопроводности с дробной производной Caputo

\title{
МАТЕМАТИКА
}

\author{
УДК 519.642
}

DOI 10.21779/2542-0321-2017-32-2-39-46

\section{Ф.Ф. Малиева}

\section{Об устойчивости разностной схемы с весами для уравнения теплопроводности с дробной производной Caputo}

Дагестанский государственный университет; Россия, 367001, г. Махачкала, 43a; faridadavudova@mail.ru

Для решения дифференциальных уравнений дробного порядка используются как аналитические, так и численные методы. В связи с большими трудностями, возникающими при поиске аналитических решений уравнений с дробными производными, в этой статье предпринимается попытка разработки численного метода его решения.

Унифицированным методом приближенного решения дифференциальных уравнений, применимым для широкого класса уравнений математической физики, является метод конечных разностей (или метод сеток). Еще одним достоинством данного метода является простота его реализации и универсальность получаемых программ.

В методе конечных разностей осуществляется переход от непрерывной среды к некоторой ее дискретной модели. При таком переходе должны сохраняться основные свойства физического процесса, прежде всего законы сохранения (тепла, массы, энергии и т. д.).

В работе исследована краевая задача для уравнения теплопроводности с производными дробного порядка. Получены условия устойчивости разностной схемы с весами для уравнения теплопроводности с производными дробного порядка.

Ключевые слова: дробная производная, аппроксимация, разностная схема, устойчивость, сходимость.

Численным методам решения дифференциальных уравнений с производными дробного порядка посвящены работы [3-10].

\section{Краевая задача для уравнения теплопроводности с дробной производной Сарuto}

Рассмотрим следующую краевую задачу для уравнения теплопроводности с оператором дробного дифференцирования.

Задача. В области $D=\{(x, t): 0<x<R, 0<t<T\}$ найти решение уравнения

$$
\partial_{0 t}^{\alpha} u(x, t)=C(x, t) \frac{\partial^{2} u(x, t)}{\partial x^{2}}+f(x, t),
$$

удовлетворяющее начальному условию: $u(x, 0)=\psi(x)$ и краевым условиям:

$$
u(0, t)=\mu_{1}(t), u(R, t)=\mu_{2}(t),
$$


где $0<\alpha \leq 1, C(x, t) \geq 0$. Здесь $u(x, t)$ - температура, $C(x, t) \geq 0$ - коэффициент теплопроводности, $f(x, t)$ - удельная плотность тепловыделения за счет внутренних источников, $\partial_{0 t}^{\alpha} u(x, t)=\frac{1}{\Gamma(1-\alpha)} \int_{0}^{t} \frac{u^{\prime}(x, s)}{(t-s)^{\alpha}} d s-$ дробная производная Caputo [1].

Численный метод решения краевой задачи. Решение задачи (1), (2) найдем численным методом. Для этого в области $\bar{D}=\{(x, t): 0 \leq x \leq R, 0 \leq t \leq T\}$ введем сетку $\quad \bar{W}=\left\{\left(x_{m}, t_{n}\right): x_{m}=m h, t_{n}=n \tau, m=0,1, \ldots M, h=\frac{R}{M}, n=0,1,2, \ldots, N, \tau=\frac{T}{N}\right\} \mathrm{c}$ шагом $h$ по $x$ и $\tau$ по $t$.

Для производной дробного порядка имеет место следующая разностная аппроксимация [6]:

$$
\begin{aligned}
& \left(\partial_{0 t}^{\alpha} u(x, t)\right)_{n}= \\
& =\frac{1}{\Gamma(2-\alpha)} \sum_{k=1}^{n} \frac{u\left(x, t_{k+1}\right)-u\left(x, t_{k}\right)}{\tau^{\alpha}} \cdot\left((n-k+1)^{1-\alpha}-(n-k)^{1-\alpha}\right)+R_{n},
\end{aligned}
$$

где

$$
\begin{aligned}
& R_{n}=\frac{1}{\Gamma(1-\alpha)} \sum_{k=1}^{n} \int_{(k-1) \tau}^{k \tau} \frac{u_{s s}(x, \xi)\left(s-t_{k}\right)}{\left(t_{n}-s\right)^{\alpha}} d s \leq \frac{M \tau}{\Gamma(1-\alpha)} \sum_{k=1}^{n} \int_{t_{k-1}}^{t_{k}} \frac{d s}{\left(t_{n}-s\right)^{\alpha}}= \\
& =\frac{M \tau}{\Gamma(2-\alpha)} \sum_{k=1}^{n}\left(t_{n-k+1}^{1-\alpha}-t_{n-k}^{1-\alpha}\right)=\frac{M t_{n}^{1-\alpha} \tau}{\Gamma(2-\alpha)}=O(\tau),
\end{aligned}
$$

$\xi-$ некоторая промежуточная точка между $S$ и $t_{k}$.

А для частной производной $\frac{\partial^{2} u(x, t)}{\partial x^{2}}$ имеет место аппроксимация:

$$
\left(\frac{\partial^{2} u(x, t)}{\partial x^{2}}\right)_{m}=\frac{u\left(x_{m+1}, t\right)-2 u\left(x_{m}, t\right)+u\left(x_{m-1}, t\right)}{h^{2}}+O\left(h^{2}\right) .
$$

Воспользовавшись разностными аппроксимациями (3) и (4), получим разностную задачу с весами

$$
\begin{gathered}
\frac{1}{\Gamma(2-\alpha) \tau^{\alpha}} \sum_{k=0}^{n+1}\left(u_{m}^{k+1}-u_{m}^{k}\right)\left((n-k+1)^{1-\alpha}-(n-k)^{1-\alpha}\right)=C_{m}^{n}\left(\sigma \Lambda u_{m}^{n+1}+(1-\sigma) \Lambda u_{m}^{n}\right)+\varphi_{m}^{n}, \\
u_{0}^{n}=\mu_{1}\left(t_{n}\right), u_{m}^{n}=\mu_{2}\left(t_{n}\right), u_{m}^{0}=\psi\left(x_{m}\right),
\end{gathered}
$$

где $C_{m}^{n}=C\left(x_{m}, t_{n}\right), f_{m}^{n}=f\left(x_{m}, t_{n}\right)$,

$$
\Lambda u_{m}^{n+1}=\frac{u_{m+1}^{n+1}-2 u_{m}^{n+1}+u_{m-1}^{n+1}}{h^{2}}, \Lambda u_{m}^{n}=\frac{u_{m+1}^{n}-2 u_{m}^{n}+u_{m-1}^{n}}{h^{2}}
$$


Разностную схему (5) запишем в операторном виде:

$$
\begin{aligned}
& \left(E+\sigma \cdot \Gamma(2-\alpha) \tau^{\alpha} A\right) u^{n+1}=\left(B+(1-\sigma) \Gamma(2-\alpha) \tau^{\alpha} A\right) u^{n}+\Gamma(2-\alpha) \tau^{\alpha} \varphi^{n}- \\
& -\sum_{k=1}^{n-2}\left(u^{k+1}-u^{k}\right)\left((n-k+1)^{1-\alpha}-(n-k)^{1-\alpha}\right),
\end{aligned}
$$

где $u^{n}=\left(u_{1}^{n}, u_{2}^{n}, \ldots, u_{N-1}^{n}\right), \varphi^{n}=\left(\varphi_{1}^{n}, \varphi_{2}^{n}, \ldots, \varphi_{N-1}^{n}\right)^{T}$.

В формуле (7) $\left(u_{0}\left(x_{1}\right), u_{0}\left(x_{2}\right), \ldots, u_{0}\left(x_{N-1}\right)\right)^{T},(A u)_{m}=-C_{m}^{n} \frac{u_{m+1}-2 u_{m}+u_{m-1}}{h^{2}}$,

$$
B=\left(\begin{array}{lrrrr}
2-2^{1-\alpha} & 0 & 0 & \ldots & 0 \\
0 & 2-2^{1-\alpha} & 0 & \ldots & 0 \\
\ldots \ldots \ldots \ldots \ldots \ldots \ldots \ldots \ldots \ldots & \ldots \ldots \ldots \ldots \ldots \ldots \\
0 & 0 & 0 & 2-2^{1-\alpha}
\end{array}\right) .
$$

\section{Устойчивость разностной схемы по начальным данным}

Разрешимость уравнения (7) относительно $u^{n+1}$ эквивалентна обратимости оператора $E+\sigma \cdot \Gamma(2-\alpha) \tau^{\alpha} A$. Оператор $E+\sigma \cdot \Gamma(2-\alpha) \tau^{\alpha} A$ будет иметь обратный, если потребовать $1+\sigma \cdot \Gamma(2-\alpha) \tau^{\alpha} \lambda_{k}>0, k=1,2, \ldots, N-1$, где $\lambda_{k}>0$ - собственные числа оператора $A[2]$.

При каждом $n$ решении $u_{m}^{n}=u\left(x_{m}, t_{n}\right)$ можно представить в виде

$$
u\left(x_{m}, t_{n}\right)=\sum_{s=1}^{M-1} c_{s}\left(t_{n}\right) \mu_{s}\left(x_{m}\right) \text {. }
$$

Правая часть $\varphi_{m}^{n}$ уравнения (7) также допускает разложение

$$
\varphi\left(x_{m}, t_{n}\right)=\sum_{s=1}^{M-1} \hat{\varphi}_{s}\left(t_{n}\right) \mu_{s}\left(x_{m}\right) .
$$

Здесь $c_{s}\left(t_{n}\right)$ и $\hat{\varphi}_{s}\left(t_{n}\right)$ - коэффициенты Фурье функций $u\left(x_{m}, t_{n}\right), \varphi\left(x_{m}, t_{n}\right)$ соответственно. Подставляя (8), (9) в уравнение (7) и учитывая, что $\left(\mu_{s}(x)\right)_{\overline{x x}, m}=-\lambda_{s} \mu_{s}\left(x_{m}\right)$, получим

$$
\begin{aligned}
& \sum_{s=1}^{M-1} \mu_{s}\left(x_{m}\right)\left[\sum_{k=0}^{n+1} \frac{c_{s}\left(t_{k+1}\right)-c_{s}\left(t_{k}\right)}{\Gamma(2-\alpha) \tau^{\alpha}}\left((n-k+1)^{1-\alpha}-(n-k)^{1-\alpha}\right)+\sigma \lambda_{s} c_{s}\left(t_{n+1}\right)+\right. \\
& \left.+(1-\sigma) \lambda_{s} c_{s}\left(t_{n}\right)-\hat{\varphi}_{s}\left(t_{n}\right)\right]=0
\end{aligned}
$$

Функции $\mu_{s}(x)$ линейно независимые. Следовательно, 


$$
\begin{aligned}
& \left(1+\sigma \cdot \Gamma(2-\alpha) \tau^{\alpha} \lambda_{s}\right) c_{s}\left(t_{n+1}\right)-\left(\left(2-2^{1-\alpha}\right)+(1-\sigma) \Gamma(2-\alpha) \tau^{\alpha} \lambda_{s}\right) c_{s}\left(t_{n}\right)+ \\
& +\sum_{k=1}^{n-2}\left(c_{s}\left(t_{k+1}\right)-c_{s}\left(t_{k}\right)\right)\left((n-k+1)^{1-\alpha}-(n-k)^{1-\alpha}\right)-\Gamma(2-\alpha) \tau^{\alpha} \hat{\varphi}_{s}\left(t_{n}\right)=0
\end{aligned}
$$

$n=0,1, \ldots, N-1, S=1,2, \ldots, M-1$.

Уравнение (10) при каждом $s$ представляет собой разностное уравнение относительно $c^{(n)}=c_{S}\left(t_{n}\right)$. Чтобы найти единственное решение, зададим начальное условие $c_{s}(0)=\left(u^{0}, \mu_{s}\right)$.

Из уравнения (10) получим

$$
\begin{aligned}
& c_{s}\left(t_{n+1}\right)=\frac{\left(2-2^{1-\alpha}\right)-(1-\sigma) \Gamma(2-\alpha) \tau^{\alpha} \lambda_{s}}{1+\sigma \cdot \Gamma(2-\alpha) \tau^{\alpha} \lambda_{s}} c_{s}\left(t_{n}\right)+\frac{\Gamma(2-\alpha) \tau^{\alpha}}{1+\sigma \cdot \Gamma(2-\alpha) \tau^{\alpha} \lambda_{s}} \hat{\varphi}_{s}\left(t_{n}\right)- \\
& -\frac{1}{1+\sigma \cdot \Gamma(2-\alpha) \tau^{\alpha} \lambda_{s}} \sum_{k=1}^{n-2}\left(c_{s}\left(t_{k+1}\right)-c_{s}\left(t_{k}\right)\right)\left((n-k+1)^{1-\alpha}-(n-k)^{1-\alpha}\right) .
\end{aligned}
$$

Учитывая (8), (11) решение $u_{m}^{n+1}$ задачи запишем в виде

где

$$
\begin{gathered}
u_{m}^{n+1}=\sum_{s=1}^{M-1}\left[p_{s} \cdot c_{s}\left(t_{n}\right)+q_{s} \cdot \hat{\varphi}_{s}\left(t_{n}\right)-\frac{1}{1+\sigma \cdot \Gamma(2-\alpha) \tau^{\alpha} \lambda_{s}} \sum_{k=2}^{n+1}\left(\Delta_{\tau}^{\alpha} c_{s}\left(t_{n}\right)\right)\right] \mu_{s}\left(x_{m}\right), \\
p_{s}=\frac{\left(2-2^{1-\alpha}\right)-(1-\sigma) \Gamma(2-\alpha) \tau^{\alpha} \lambda_{s}}{1+\sigma \cdot \Gamma(2-\alpha) \tau^{\alpha} \lambda_{s}}, q_{s}=\frac{\Gamma(2-\alpha) \tau^{\alpha}}{1+\sigma \cdot \Gamma(2-\alpha) \tau^{\alpha} \lambda_{s}}, \\
\Delta_{\tau}^{\alpha} c_{s}\left(t_{n}\right)=\sum_{k=1}^{n-2}\left(c_{s}\left(t_{k+1}\right)-c_{s}\left(t_{k}\right)\right)\left((n-k+1)^{1-\alpha}-(n-k)^{1-\alpha}\right) .
\end{gathered}
$$

Обозначая

$$
\begin{gathered}
\bar{u}_{m}^{n+1}=\sum_{s=1}^{M-1}\left[p_{s} \cdot c_{s}\left(t_{n}\right)\right] u_{s}\left(x_{m}\right), \tilde{u}_{m}^{n+1}=\sum_{s=1}^{M-1}\left[q_{s} \cdot \hat{\varphi}_{s}\left(t_{n}\right)\right] \mu_{s}\left(x_{m}\right), \\
=n+1 \\
u_{m}=-\sum_{s=1}^{M-1} \frac{1}{1+\sigma \cdot \Gamma(2-\alpha) \tau^{\alpha} \lambda_{s}} \cdot \Delta_{\tau}^{\alpha} c_{s}\left(t_{n}\right) \cdot \mu_{s}\left(x_{m}\right),
\end{gathered}
$$

получим, что

$$
u_{m}^{n+1} \leq \bar{u}_{m}^{n+1}+\widetilde{u}_{m}^{n+1}+\bar{u}_{m}^{n+1} .
$$

В силу ортонормированности базиса $\left\{\mu_{s}\right\}$ получаем

$$
\left\|\bar{u}^{n+1}\right\|^{2}=\sum_{m=1}^{M-1}\left(\bar{u}_{m}^{n+1}\right)^{2} h=\sum_{s=1}^{M-1} p_{s}^{2} \cdot\left(c_{s}\left(t_{n}\right)\right)^{2} .
$$

Следовательно,

$$
\left\|\bar{u}^{n+1}\right\| \leq\left(\sum_{s=1}^{M-1}\left(c_{s}\left(t_{n}\right)\right)^{2}\right)^{\frac{1}{2}} \max \left|p_{s}\right|=\left\|u^{n}\right\| \max _{1 \geq S \leq M-1}\left|p_{s}\right| .
$$

Потребуем выполнения условия 


$$
\left|p_{s}\right|=\left|\frac{\left(2-2^{1-\alpha}\right)-(1-\sigma) \Gamma(2-\alpha) \tau^{\alpha} \lambda_{s}}{1+\sigma \cdot \Gamma(2-\alpha) \tau^{\alpha} \lambda_{s}}\right| \leq 1, s=1,2, \ldots, M-1 .
$$

Условие (12) эквивалентно условию

$$
\sigma \geq \frac{1}{2}-\frac{3-2^{1-\alpha}}{2 \Gamma(2-\alpha) \tau^{\alpha} \lambda_{s}}
$$

Из (13) при любом $s=1,2, \ldots, M-1$ следует неравенство:

$$
1+\sigma \cdot \Gamma(2-\alpha) \tau^{\alpha} \lambda_{s} \geq \frac{\Gamma(2-\alpha) \tau^{\alpha} \lambda_{s}}{2}+\frac{2^{1-\alpha}-1}{2}>0 .
$$

Итак, если выполнено (13), то справедлива оценка

$$
\left\|\bar{u}^{n+1}\right\| \leq\left\|u^{n}\right\| .
$$

Это означает устойчивость разностной схемы по начальным данным. Если в уравнении $F_{m}^{n}=0, \quad$ то $u_{m}^{n+1}=\bar{u}_{m}^{n+1}$. Тогда $\quad$ согласно (13) получаем $\left\|u^{n+1}\right\| \leq\left\|u^{n}\right\| \leq\left\|u^{n-1}\right\| \leq \cdots \leq\left\|u^{0}\right\|$, что означает устойчивость схемы по начальным данным в норме $\|u\|=\left(\sum_{m=1}^{M-1} h u_{m}^{2}\right)^{\frac{1}{2}}$.

Устойчивость по правой части и сходимость. Оценим функцию

$$
\tilde{u}_{m}^{n+1}=\sum_{s=1}^{M-1}\left[q_{s} \hat{\varphi}_{s}\left(t_{n}\right)\right] \mu_{s}\left(x_{m}\right) .
$$

Для этого запишем условие (3) в виде

$$
\sigma \geq \frac{1}{2}-\frac{(1-\varepsilon)\left(3-2^{1-\alpha}\right)}{\tau^{\alpha} \Gamma(2-\alpha) \tau^{\alpha} \lambda_{N-1}},
$$

где $0<\varepsilon<1, s=1,2, \ldots, M-1$. Тогда получим

$$
\begin{aligned}
& 1+\sigma \cdot \Gamma(2-\alpha) \tau^{\alpha} \lambda_{s} \geq \frac{\Gamma(2-\alpha) \tau^{\alpha} \lambda_{s}}{2}+1-\frac{(1-\varepsilon)\left(3-2^{1-\alpha}\right) \lambda_{s}}{2 \lambda_{N-1}}> \\
& >1-\frac{(1-\varepsilon)\left(3-2^{1-\alpha}\right)}{2}>1-\frac{(1-\varepsilon) \cdot 2}{2}=\varepsilon>0 .
\end{aligned}
$$

Из равенства (15) получаем

$$
\begin{aligned}
& \left\|\tilde{u}^{n+1}\right\|^{2} \leq \sum_{s=1}^{M-1}\left[\left(q_{s}\right)^{2}\left(\hat{\varphi}_{s}\left(t_{n}\right)\right)^{2}\right] \leq\left(\frac{\Gamma(2-\alpha) \tau}{\left(x_{2}^{1-\alpha}-x_{1}^{1-\alpha}\right)+\left(3 x_{1}^{1-\alpha}-x_{2}^{1-\alpha}\right) \varepsilon}\right)^{2} \sum_{s=1}^{M-1}\left(\hat{\varphi}_{s}\left(t_{n}\right)\right)^{2} \leq \\
& \leq\left(\frac{\tau}{\varepsilon}\right)^{2} \sum_{s=1}^{M-1}\left(\hat{\varphi}_{s}\left(t_{n}\right)\right)^{2} .
\end{aligned}
$$

Следовательно,

$$
\left\|\widetilde{u}^{n+1}\right\| \leq \frac{\tau}{\varepsilon}\left\|\varphi^{n}\right\|
$$


Теперь оценим функцию

$$
\begin{aligned}
& \stackrel{u}{m}_{m}^{n+1}=-\sum_{s=1}^{M-1} \frac{1}{1+\sigma \cdot \Gamma(2-\alpha) \tau^{\alpha} \lambda_{s}} \cdot \Delta_{\tau}^{\alpha} C_{s}\left(t_{n}\right) \cdot \mu_{s}\left(x_{m}\right) . \\
& \left\|=u^{n+1}\right\|^{2} \leq \sum_{s=1}^{M-1}\left(\frac{1}{1+\sigma \cdot \Gamma(2-\alpha) \tau^{\alpha} \lambda_{s}}\right)^{2} \cdot\left(\Delta_{\tau}^{\alpha} c_{s}\left(t_{n}\right)\right)^{2} \text {. }
\end{aligned}
$$

Тогда

$$
\begin{aligned}
& \|=n+1\| \leq \frac{1}{\varepsilon} \cdot\left\|\Delta_{\tau}^{\alpha} C_{S}\left(t_{n}\right)\right\| . \\
& \left\|\Delta_{\tau}^{\alpha} c_{s}\left(t_{n}\right)\right\| \leq \sum_{k=1}^{n-2}\left\|\left(c_{s}\left(t_{k+1}\right)-c_{s}\left(t_{k}\right)\right)\right\|\left((n-k+1)^{1-\alpha}-(n-k)^{1-\alpha}\right) \leq \\
& \leq r \cdot \tau \sum_{k=1}^{n-2}\left((n-k+1)^{1-\alpha}-(n-k)^{1-\alpha}\right) \leq r \cdot \tau \cdot\left(n^{1-\alpha}-2^{1-\alpha}\right) \leq r \cdot \tau \cdot n^{1-\alpha}= \\
& =r \cdot \tau \cdot\left(\frac{T}{\tau}\right)^{1-\alpha}=r \cdot T^{1-\alpha} \cdot \tau^{\alpha}=g \cdot \tau^{\alpha},
\end{aligned}
$$

где $r=\max \left|c_{s}^{\prime}(t)\right|=$ const, $g=r \cdot T^{1-\alpha}=$ const .

Следовательно,

Используя свойство нормы

$$
\left\|=u^{=n+1}\right\| \leq \frac{g \tau^{\alpha}}{\varepsilon}
$$

$$
\left\|u^{n+1}\right\| \leq\left\|\bar{u}^{n+1}\right\|+\left\|\widetilde{u}^{n+1}\right\|+\left\|u^{n+1}\right\|
$$

и оценки $(14),(17)$ и (18), получаем неравенство

$$
\left\|u^{n+1}\right\| \leq\left\|u^{n}\right\|+\frac{\tau}{\varepsilon}\left\|\varphi^{n}\right\|+\frac{g \tau^{\alpha}}{\varepsilon} .
$$

Неравенство (19) справедливо при $n=0,1, \ldots, N-1$. Суммируя (19) по $n$, получим оценку

$$
\left\|u^{n+1}\right\| \leq\left\|u^{0}\right\|+\frac{\tau}{\varepsilon} \sum_{j=0}^{n}\left\|\varphi^{j}\right\|+\frac{g \tau^{\alpha}}{\varepsilon} .
$$

Из оценки (20) следует устойчивость задачи (5) и (6) по начальным данным и по правой части.

Учитывая условие $\pi \leq T$, из оценки (20) получим

$$
\left\|u^{n+1}\right\| \leq\left\|u^{0}\right\|+\frac{T}{\varepsilon} \max _{0 \leq j \leq n}\left\|\varphi^{j}\right\|+\frac{g \tau^{\alpha}}{\varepsilon} .
$$

Таким образом, если выполнено условие (16), то разностная задача (5), (6) устойчива по начальным данным и по правой части. 


\section{Литература}

1. Нахушев А.М. Элементы дробного исчисления и их применение. - Нальчик, 2003. - 299 c.

2. Самарский А.А., Гулин А.В. Численные методы. - М.: Наука, 1989. - 430 с.

3. Meerschaert M.M., Tadjeran C. Finite difference approximations for two-sides space-fractional partial differential equations // Applied Numerical Mathematics. - 2006. № 56. - P. 80-90.

4. Бейбалаев В.Д. Математическая модель переноса в средах с фрактальной структурой // Математическое моделирование. - 2009. - Т. 21, № 5. - С. 55-62.

5. Назаралиев М.А., Бейбалаев В.Д. Численные методы решения краевой задачи для уравнения теплопереноса с производной дробного порядка // Вестник ДГУ. 2008. - Вып. 6. - С. 46-53.

6. Бейбалаев В.Д. Разностные методы решения задачи Коши для обыкновенного дифференциального уравнения с оператором дробного дифференцирования // Вестник ДГУ. - 2014. - Вып. 6. - С. 53-61.

7. Бейбалаев В.Д. Численный метод решения задачи переноса с двусторонней производной дробного порядка // Вестник СамГТУ. Сер.: Физико-математические науки. - 2009. - № 1 (18). - С. 267-270.

8. Бейбалаев В.Д. Численный метод решения математической модели теплопереноса в средах с фрактальной структурой // Фундаментальное исследование. - 2007. - № 12. - С. 67-71.

9. Лафишева М.М., Шхануков-Лафишев М.Х. Локально-одномерная разностная схема для уравнения диффузии дробного порядка // Вычислительная математика и математическая физика. - 2008. - Т. 48, № 10. - С. 1878-1887.

10. Алиханов A.A. Разностные методы решения краевых задач для волнового уравнения с дробной производной по времени // Вестник Сам. ГТУ. Сер.: Физ.-мат. науки. - 2008. - № 2 (17). - С. 13-20.

Поступила в редакиию 30 марта 2017 г.

UDC 519.642

DOI 10.21779/2542-0321-2017-32-2-39-46

\section{On the stability of a difference scheme with weights for the heat equation with a fractional derivative of Caputo}

\section{F.F. Malieva}

Dagestan State University, Russia, 367001, Makhachkala, M. Gadzhiyev St., 43a; faridadavudova@mail.ru 
Currently, both analytical and numerical methods are used for solutions of differential equations of fractional order. Due to the great difficulties in finding the analytical solutions of equations with fractional derivatives, this paper attempts to develop a numerical method of its decision.

The unified method of approximate solution of differential equations applicable to a wide class of mathematical physics equations is the finite difference method (or the method of grids). Another advantage of this method is the ease of implementation and flexibility of derived programs.

The method of finite differences uses transition from continuum to some of its discrete model. When this method is used the basic properties of the physical process are preserved, first of all, the laws of conservation (heat, mass, energy, etc.).

The article studies the boundary value problem for the heat equation with fractional order derivatives. The conditions of stability of a difference scheme with weights for the heat equation with fractional order derivatives are obtained.

Keywords: fractional derivative, approximation, difference scheme, stability, convergence.

Received 30 March, 2017 